\title{
Discrete Isoperimetric Inequalities
}

\author{
Fan Chung
}

\section{Contents}

1. Introduction 53

2. Combinatorial and normalized Laplacian $\quad 55$

3. Eigenvalues and spanning trees $\quad 57$

4. A discrepancy inequality $\quad 58$

5. Cheeger inequalities $\quad 62$

6. Diameter-eigenvalue inequalities $\quad 65$

7. Sobolev constants and Sobolev inequalities 67

8. Harnack inequalities 68

9. Heat kernel eigenvalue inequalities $\quad 69$

$\begin{array}{ll}\text { 10. Paths and cycles } & 71\end{array}$

11. Universal graphs for trees of bounded degrees 72

12. Chromatic numbers and list chromatic numbers $\quad 75$

$\begin{array}{ll}\text { 13. Turán numbers } & 76\end{array}$

14. Random walks and contingency tables $\quad 76$

$\begin{array}{lr}\text { 15. Concluding remarks } & 79\end{array}$

$\begin{array}{lr}\text { References } & 80\end{array}$

\section{Introduction}

One of the earliest problems in geometry is the isoperimetric problem, which was considered by the ancient Greeks. The problem is to find, among all closed curves of a given length, the one which encloses the maximum area. Isoperimetric problems for the discrete domain are in the same spirit but with different complexity. A basic model for communication and computational networks is a graph $G=(V, E)$ consisting of a set $V$ of vertices and a prescribed set $E$ of unordered pairs of vertices. For a subset $X$ of vertices, there are two types of boundaries:

- The edge boundary $\partial(X)=\{\{u, v\} \in E: u \in X, v \in V \backslash X\}$.

- The vertex boundary $\delta(X)=\{v \in V \backslash X:\{v, u\} \in E$ for some $u \in X\}$.

Numerous questions arise in examining the relations between $\partial(X), \delta(X)$ and the sizes of $X$. Here the size of a subset of vertices may mean the number of 
vertices, the number of incident edges, or some other appropriate measure defined on graphs.

In this paper, we will survey spectral techniques for studying discrete isoperimetric inequalities and the like. In addition, a number of applications in extremal graph theory and random walks will be included. This paper is organized as follows:

Section 1: A brief introduction and an outline are given.

Section 2: Definitions and notations of the combinatorial Laplacian and normalized Laplacian are introduced as well as the historical matrix-tree theorem.

Section 3: Enumeration of spanning trees using eigenvalues of the combinatorial and normalized Laplacians

Section 4: Complimentary to the isoperimetric inequalities is the discrepancy inequalities. Instead of tracking the number of edges leaving $X$, discrepancy inequalities provide estimates of the edges remaining in $X$. Here we give several versions of discrepancy inequalities for general graphs.

Section 5: The usual Cheeger inequality is for bounding the edge boundary. Here we also consider vertex boundaries and their generalizations.

Section 6: Eigenvalues are related to the diameter of a graph, i.e., the maximum distance between any two vertices. We will give several eigenvalue inequalities for bounding the diameter and the distance between two or more subsets both for graphs and manifolds.

Section 7: We consider discrete Sobolev inequalities which include Cheeger inequalities as a special case.

Section 8: Harnack inequalities, which provide pointwise estimates for eigenfunctions, can be established for certain convex subgraphs of homogeneous graphs.

Section 9: The heat kernel of a graph contains all the spectral information about the graph. For example, the heat kernel can be used to deduce bounds for eigenvalues of certain induced subgraphs.

Section 10-14: The containment or avoidance of specified subgraphs, such as paths, cycles and cliques of given sizes are central themes in classical Ramsey and Turán theory. Our focus here is to derive such extremal graph properties as direct consequences of spectral bounds. The applications include the forcing of long paths and cycles, universal graphs for trees, chromatic numbers and list chromatic numbers, and the Turán numbers, as well as random walks involving the enumeration of contingency tables.

In the applicational sections, there are some overlaps with the study of the socalled $(n, d, \lambda)$-graphs (i.e., regular graphs on $n$ vertices having degree $d$ with all but one eigenvalue of the adjacency matrix bounded above by $\lambda$ ). Such graphs are extensively examined in many papers by Alon $[\mathbf{4}]$ and others $[\mathbf{4 6}, \mathbf{6 0}]$. There is a recent comprehensive survey by Krivelevich and Sudakov [46] on $(n, d, \lambda)$-graphs. Here we deal with general graphs with no degree constraints.

Throughout the paper, we consider only finite graphs. The reader is referred to the book by Woess [63], which explores isoperimetric properties and random walks on infinite graphs. There is a close connection between discrete isoperimetric inequalities and their continuous counterpart, as evidenced in Section 7 to 9 . Isoperimetric inequalities for Riemannian geometry have been long studied and well developed (see $[\mathbf{1 2}, \mathbf{6 4}]$ ). As a result, one of the earlier approaches on discrete 
isoperimetric inequalities focuses on discretizations of manifolds $[35,42]$. Another approach is to study graphs with group symmetry [58] or random walks on finite groups $[\mathbf{3 6}]$. In this paper, we consider general graphs and our approach here is from a graph-theoretic point of view.

\section{Combinatorial and normalized Laplacian}

One of the classical results in graph theory is the matrix-tree theorem by Kirchhoff $[\mathbf{4 3}]$, which states that the number of spanning trees in a graph is determined by the determinant of a principle minor of the combinatorial Laplacian. For a graph $G$ with vertex set $V$ and edge set $E$, the combinatorial Laplacian $L$ is a matrix with rows and columns indexed by vertices in $V$ and can be written as

$$
L=D-A
$$

where $D$ is the diagonal matrix with $D(v, v)$ equal to the degree $d_{v}$ of $v$ and $A$ is the adjacency matrix of $G$. If $G$ is a simple graph with no loops or multiple edges, $A(u, v)=1$ if $u$ and $v$ are adjacent or else $A(u, v)=0$. In this paper, we restrict ourselves to simple graphs although most of the statements and results can be easily carried out to general graphs or weighted graphs.

The combinatorial Laplacian has its root in homological algebra and spectral geometry. We can write $L$ as

$$
L=B B^{*}
$$

where $B$ is the incidence matrix whose rows are indexed by the vertices and whose columns are indexed by the edges of $G$ such that each column corresponding to an edge $e=\{u, v\}$ has an entry 1 in the row corresponding to $u$, an entry -1 in the row corresponding to $v$, and has zero entries elsewhere. (As it turns out, the choice of signs can be arbitrary as long as one is positive and the other is negative.) Also, $B^{*}$ denotes the transpose of $B$. Here $B$ can be viewed as a "boundary operator" mapping "1-chains" defined on edges (denoted by $C_{1}$ ) of a graph to "0-chains" defined on vertices (denoted by $C_{0}$ ). Then, $B^{*}$ is the corresponding "coboundary operator" and we have

$$
C_{1} \stackrel{B}{\longleftarrow} C_{B^{*}}
$$

This fact can be used to give a short proof of the matrix-tree theorem as follows: For a fixed vertex $v$, let $L^{\prime}$ denote the submatrix obtained by deleting the $v$ th row and $v$ th column of $L$. Since $L=B B^{*}$, we have $L^{\prime}=B_{0} B_{0}^{*}$ where $B_{0}$ denotes the submatrix of $B$ without the $v$ th column. By the Binet-Cauchy Theorem [53] we have

$$
\operatorname{det} B_{0} B_{0}^{*}=\sum_{X} \operatorname{det} B_{X} \operatorname{det} B_{X}^{*}
$$

where the sum ranges over all possible choices of size $n-1$ subsets $X$ of $E(G)$ and $B_{X}$ denotes the square submatrix of $B_{0}$ whose $n-1$ columns correspond to the edges in $X$ and whose rows are indexed by all the vertices except for $v$. Standard graph-theoretical arguments can be used to show that $\left|\operatorname{det} B_{X}\right|=1$ if edges in $X$ form a tree and 0 otherwise. Thus, $\operatorname{det} B_{0} B_{0}^{*}$ is exactly the number of spanning trees in $G$, as asserted by the matrix-tree theorem. 
Geometrically, the combinatorial Laplacian $L$ can be viewed as the discrete ana$\log$ of of the Laplace-Beltrami operator, especially for graphs that are the Cartesian products of paths, for example. For a path $v_{0}, v_{1}, \ldots, v_{n}$ and a function $f$ that assigns a real value to each $v_{i}$, the combinatorial Laplacian can be written as :

$$
\begin{aligned}
L f(v) & =\sum_{u \sim v}(f(v)-f(u)) \\
L f\left(v_{i}\right) & =\left(f\left(v_{i}\right)-f\left(v_{i-1}\right)\right)-\left(f\left(v_{i+1}\right)-f\left(v_{i}\right)\right) \\
& =\nabla f\left(v_{i}, v_{i-1}\right)-\nabla f\left(v_{i+1}, v_{i}\right) \\
& =\Delta f\left(v_{i}\right)
\end{aligned}
$$

for $0<i<n$. Here we use the following notation:

$$
\begin{aligned}
\text { For an edge }\{u, v\}, \nabla f(u, v) & =f(u)-f(v) . \\
\text { For a vertex } v, \Delta f(v) & =\sum_{u \sim v}(f(v)-f(u)) . \\
\|\nabla f\|^{2}=\sum_{u \sim v}(f(u)-f(v))^{2} & =\langle f, L f\rangle .
\end{aligned}
$$

where $u \sim v$ means $\{u, v\} \in E(G)$. Note that $\nabla f(u, v)$ can be viewed as the first derivative in the direction of the edge $\{u, v\}$ and $\Delta f(v)$ can be regarded as the sum of second derivatives over all directions along the edges incident to $v$. The so-called Dirichlet sum is just $\|\nabla f\|^{2}$ as indicated above.

Since $L$ is self-adjoint as seen in (1), its eigenvalues are non-negative. We denote the eigenvalues of $L$ of a graph $G$ on $n$ vertices by

$$
0=\sigma_{0} \leq \sigma_{1} \leq \ldots \leq \sigma_{n-1} .
$$

One of the main approaches in spectral graph theory is to deduce various graph properties from eigenvalue distributions. In order to so so, it is sometimes appropriate to consider the normalized Laplacian $\mathcal{L}$, especially for diffusion-type problems such as random walks.

$$
\mathcal{L}=D^{-1 / 2} L D^{-1 / 2}
$$

Here we preclude isolated vertices in order to guarantee that $D$ is invertible, an inconsequential constraint in practice. For regular graphs $L$ and $\mathcal{L}$ are basically the same (up to a scale factor). However, for general graphs, it is often advantageous to utilize the normalized Laplacian. We denote the eigenvalues of $\mathcal{L}$ of $G$ by

$$
0=\lambda_{0} \leq \lambda_{1} \leq \ldots \leq \lambda_{n-1} .
$$

To compare $\mathcal{L}$ and $L$, we note that

$$
\lambda_{1}=\inf _{f \perp \phi_{0}} \frac{\langle f, \mathcal{L} f\rangle}{\langle f, f\rangle}=\inf _{\sum g(x) d_{x}=0} \frac{\langle g, L g\rangle}{\langle g, D g\rangle}=\inf _{\sum g(x) d_{x}=0} \frac{\|\nabla g\|^{2}}{\langle g, D g\rangle}
$$

where $\phi_{0}$ denotes the eigenfunction associated with eigenvalue 0 of $\mathcal{L}$ and $g=$ $D^{-1 / 2} f$. In contrast,

$$
\sigma_{1}=\inf _{\sum_{x} f(x)=0} \frac{\langle f, L f\rangle}{\langle f, f\rangle}=\inf _{\sum_{x} f(x)=0} \frac{\|\nabla f\|^{2}}{\|f\|^{2}} .
$$

From the above equations, we see that $\mathcal{L}$ is the Laplace operator with the weight/measure of a vertex taken to be the degree of the vertex while $L$ is the corresponding operator having all vertices with weights equal. The combinatorial 
Laplacian is simpler, but the normalized Laplacian is sometimes better for capturing graph properties that are sensitive to degree distributions.

\section{Eigenvalues and spanning trees}

We consider separately the cases for the combinatorial Laplacian and normalized Laplacian.

3.1. Eigenvalues of the combinatorial Laplacian and spanning trees. The number of spanning trees, denoted by $\tau(G)$, can be related to the eigenvalues of $L$ in the following folklore theorem: For completeness, we briefly describe the proof here.

TheOREM 1. For a graph $G$ on $n$ vertices, the number of spanning trees $\tau(G)$ is :

$$
\tau(G)=\frac{1}{n} \prod_{i \neq 0} \sigma_{i}
$$

where $\sigma_{i}$ are eigenvalues of the combinatorial Laplacian $L$.

Proof. Consider the characteristic polynomial $p(x)$ of the combinatorial Laplacian $L$.

$$
p(x)=\operatorname{det}(L-x I) .
$$

The coefficient of the linear term is exactly

$$
-\prod_{i \neq 0} \sigma_{i}
$$

On the other hand, the coefficient of the linear term of $p(x)$ is -1 times the sum of the determinant of $n$ principle submatrices of $L$ obtained by deleting the $i$ th row and $i$ th column. By the matrix-tree theorem, the product $\prod_{i \neq 0} \sigma_{i}$ is exactly $n$ times the number of spanning trees of $G$.

\subsection{Eigenvalues of the normalized Laplacian and spanning trees.}

THEOREM 2. For a graph $G$ with degree sequences $\left(d_{v}\right)$, the number of spanning trees $\tau(G)$ is

$$
\tau(G)=\frac{\Pi_{v} d_{v}}{\sum_{v} d_{v}} \Pi_{i \neq 0} \lambda_{i}
$$

where $\lambda_{i}$ are eigenvalues of the normalized Laplacian $\mathcal{L}$.

Proof. We consider the coefficient of the linear term in

$$
\mathcal{P}(x)=\operatorname{det}(\mathcal{L}-x I)
$$

We have

$$
-\prod_{i \neq 0} \lambda_{i}=-\sum_{v} \operatorname{det} \mathcal{L}_{v}
$$

where $\mathcal{L}_{v}$ is the submatrix obtained by deleting the row and column corresponding to $v$. From the matrix-tree theorem, we have

$$
\operatorname{det} \mathcal{L}_{v}=\frac{\operatorname{det} L_{v}}{\Pi_{u \neq v} d_{u}} \operatorname{det} \mathcal{L}_{v}=\frac{\tau(G)}{\Pi_{u \neq v} d_{u}} .
$$

Thus we have

$$
\tau(G)=\frac{\Pi_{v} d_{v}}{\sum_{v} d_{v}} \Pi_{i \neq 0} \lambda_{i}
$$

as desired. 


\section{A discrepancy inequality}

For a graph $G$ and a subset $X$ of vertices in $G$, the volume $\operatorname{vol}(X)$ is defined by

$$
\operatorname{vol}(X)=\sum_{v \in X} d_{v}
$$

where $d_{v}$ is the degree of $v$. We note that for a simple graph $G$, the degree $d_{v}$ of $v$ is the number of neighbors of $v$ in $G$. We will denote the volume of $G$ by $\operatorname{vol}(G)=\sum_{v} d_{v}$.

For two subsets $X$ and $Y$ of vertices in $G$, we write

$$
e(X, Y)=\{(x, y): x \in X, y \in Y,\{x, y\} \in E(G)\} .
$$

Eigenvalues can be used to estimate $e(X, Y)$, as summarized in the discrepancy inequalities in this section.

\subsection{A general discrepancy inequality.}

Theorem 3. For a graph $G$ and two subsets of vertices $X$ and $Y$, we have

$$
\left|e(X, Y)-\frac{\operatorname{vol}(X) \operatorname{vol}(Y)}{\operatorname{vol}(G)}\right| \leq \bar{\lambda} \frac{\sqrt{\operatorname{vol}(X) \operatorname{vol}(\bar{X}) \operatorname{vol}(Y) \operatorname{vol}(\bar{Y})}}{\operatorname{vol}(G)}
$$

where the normalized Laplacian has eigenvalues $\lambda_{i}$, and $\bar{\lambda}=\max _{i \neq 0}\left|1-\lambda_{i}\right|$.

Proof. We consider the characteristic function of $X$.

$$
\psi_{X}(u)= \begin{cases}1 & \text { if } u \in X, \\ 0 & \text { otherwise. }\end{cases}
$$

Then we can write

$$
\begin{aligned}
e(X, Y) & =\left\langle\psi_{X}, A \psi_{Y}\right\rangle \\
& =\left\langle\psi_{X}, D^{1 / 2}(I-\mathcal{L}) D^{1 / 2} \psi_{Y}\right\rangle \\
& =a_{0} b_{0}+\sum_{i \neq 0} a_{i} b_{i}\left(1-\lambda_{i}\right)
\end{aligned}
$$

where $D^{1 / 2} \psi_{X}=\sum_{i} a_{i} \phi_{i}, D^{1 / 2} \psi_{Y}=\sum_{i} b_{i} \phi_{i}$, and the $\phi_{i}$ 's are orthonormal eigenfunctions of $\mathcal{L}$ associated with $\lambda_{i}$. In particular, $\phi_{0}(v)=\sqrt{d_{v} / \operatorname{vol}(G)}$ and $a_{0} b_{0}=$ $\operatorname{vol}(X) \operatorname{vol}(Y) / \operatorname{vol}(G)$. Thus

$$
\begin{aligned}
\left|e(X, Y)-\frac{\operatorname{vol}(X) \operatorname{vol}(Y)}{\operatorname{vol}(G)}\right| & =\left|e(X, Y)-a_{0} b_{0}\right| \\
& =\sum_{i \neq 0} a_{i} b_{i}\left(1-\lambda_{i}\right) \\
& \leq \bar{\lambda} \sum_{i \neq 0}\left|a_{i} b_{i}\right| \\
& \leq \bar{\lambda} \sqrt{\sum_{i \neq 0} a_{i}^{2} \sum_{j \neq 0} b_{j}^{2}} \\
& =\bar{\lambda} \frac{\sqrt{\operatorname{vol}(X) \operatorname{vol}(\bar{X}) \operatorname{vol}(Y) \operatorname{vol}(\bar{Y})}}{\operatorname{vol}(G)}
\end{aligned}
$$

as desired. 
4.2. Discrepancy inequalities using combinatorial Laplacians. The discrepancy inequalities using eigenvalues of the combinatorial Laplacian are more complicated. We will give several versions of the discrepancy inequalities depending on the intersection of subsets $X$ and $Y$. We will use the fact that an eigenfunction of $L$ associated with eigenvalue 0 is the all 1's function (but it is in general not an eigenfunction for $A$ ).

THEOREM 4. Suppose that a graph $G$ with $n$ vertices has average degree $d$ and the eigenvalues $\sigma_{i}$ of the combinatorial Laplacian satisfy $\left|d-\sigma_{i}\right| \leq \theta$ for $i \neq 0$. Then for any two subsets $X$ and $Y$ of vertices in $G$, the number $e(X, Y)$ of edges with one endpoint in $X$ and the other in $Y$ satisfies

$$
\left|e(X, Y)-\frac{d}{n}\right| X|| Y|+d| X \cap Y|-\operatorname{vol}(X \cap Y)| \leq \frac{\theta}{n} \sqrt{|X|(n-|X|)|Y|(n-|Y|)}
$$

The proof of Theorem 4 will be given later. We will first state several immediate consequences of Theorem 4.

THEOREM 5. Suppose that a graph $G$ with $n$ vertices has average degree $d$ and the eigenvalues $\sigma_{i}$ of the combinatorial Laplacian satisfy $\left|d-\sigma_{i}\right| \leq \theta$ for $i \neq 0$. Then for any two disjoint subsets $X$ and $Y$ of vertices in $G$, the number $e(X, Y)$ of (ordered) edges with first endpoint in $X$ and the second endpoint in $Y$ satisfies

$$
\left|e(X, Y)-\frac{d}{n}\right| X|| Y|| \leq \frac{\theta}{n} \sqrt{|X|(n-|X|)|Y|(n-|Y|)} .
$$

Corollary 1. Suppose that a graph $G$ with $n$ vertices has average degree $d$ and the eigenvalues $\sigma_{i}$ of the combinatorial Laplacian satisfy $\left|d-\sigma_{i}\right| \leq \theta$ for $i \neq 0$. For two disjoint subsets $X$ and $Y$ each with at least $\theta n / d$ vertices in $G$, there is at least one edge joining a vertex in $X$ to a vertex in $Y$.

Proof. If $e(X, Y)=0$, we have, by substituting into (2),

$$
\frac{d}{n} \sqrt{|X||Y|} \leq \frac{\theta}{n} \sqrt{|\bar{X}||\bar{Y}|}
$$

which is impossible for $|X|,|Y| \geq \theta n / d$.

By setting $Y$ to be the complement of $X$ in (2), we have the following:

COROllary 2. Suppose that a graph $G$ with $n$ vertices has average degree $d$ and the eigenvalues $\sigma_{i}$ of the combinatorial Laplacian satisfy $\left|d-\sigma_{i}\right| \leq \theta$ for $i \neq 0$. Then for a subset $X$ of vertices and its complement $\bar{X}$, the number $e(X, \bar{X})$ of (ordered) edges with first endpoint in $X$ and the second endpoint in $\bar{X}$ satisfies

$$
\frac{d-\theta}{n}|X||\bar{X}| \leq e(X, \bar{X}) \leq \frac{d+\theta}{n}|X||\bar{X}| .
$$

By setting $Y=\{v\}$ in (2), we have the following:

Corollary 3. Suppose that a graph $G$ with $n$ vertices has average degree $d$ and the eigenvalues $\sigma_{i}$ of the combinatorial Laplacian satisfy $\left|d-\sigma_{i}\right| \leq \theta$ for $i \neq 0$. Then for all vertices $v$, we have

$$
\frac{n}{n-1}(d-\theta) \leq d_{v} \leq \frac{n}{n-1}(d+\theta)<d+\theta .
$$

Using Theorem 5, we will prove the following: 
THEOREM 6. Suppose that a graph $G$ with $n$ vertices has average degree $d$ and the eigenvalues $\sigma_{i}$ of the combinatorial Laplacian satisfy $\left|d-\sigma_{i}\right| \leq \theta$ for $i \neq 0$. Suppose that vertex $v$ has $l_{v}$ loops. Then for a subset $X$ of vertices in $G$, the number $e(X, X)$ of ordered pairs corresponding to edges with both endpoints in $X$ satisfies

$$
\left|e(X, X)-\frac{d|X|^{2}}{n}+\left(\frac{d|X|}{n}-\sum_{v \in X} l_{v}\right)\right| \leq \frac{2 \theta}{n}|X|(n-|X| / 2) .
$$

Proof. Let $x=|X|$ and $X^{\prime}$ denote a subset of $X$ of size $x^{\prime}=\lfloor x / 2\rfloor$. We apply Theorem 5 on $X^{\prime}$ and $X \backslash X^{\prime}$ and we have

$$
\left|e\left(X^{\prime}, X \backslash X^{\prime}\right)-\frac{d}{n}\left\lfloor\frac{x}{2}\right\rfloor\left\lceil\frac{x}{2}\right\rceil\right| \leq \frac{\theta}{n} \sqrt{x^{\prime}\left(n-x^{\prime}\right)\left(x-x^{\prime}\right)\left(n-x+x^{\prime}\right)} .
$$

Since

$$
\left(\begin{array}{c}
x-2 \\
x^{\prime}-1
\end{array}\right)\left(e(X, X)-\sum_{v \in X} l_{v}\right)=\sum_{X^{\prime} \subseteq X,\left|X^{\prime}\right|=x^{\prime}} e\left(X^{\prime}, X \backslash X^{\prime}\right)
$$

we have

$$
\begin{aligned}
& \left(\begin{array}{c}
x-2 \\
x^{\prime}-1
\end{array}\right)\left|e(X, X)-\frac{d}{n} x^{2}+\left(\frac{d x}{n}-\sum_{v \in X} l_{v}\right)\right| \\
= & \left|\left(\sum_{X^{\prime} \subseteq X,\left|X^{\prime}\right|=x^{\prime}} e\left(X^{\prime}, X \backslash X^{\prime}\right)-\left(\begin{array}{c}
x-2 \\
x^{\prime}-1
\end{array}\right) \frac{d}{n} x(x-1)\right)\right| \\
= & \left|\sum_{X^{\prime} \subseteq X,\left|X^{\prime}\right|=x^{\prime}}\left(e\left(X^{\prime}, X \backslash X^{\prime}\right)-\frac{d}{n}\left\lfloor\frac{x}{2}\right\rfloor\left\lceil\frac{x}{2}\right\rceil\right)\right| \\
\leq & \left(\begin{array}{c}
x \\
x^{\prime}
\end{array}\right) \frac{\theta}{n} \sqrt{x^{\prime}\left(n-x^{\prime}\right)\left(x-x^{\prime}\right)\left(n-x+x^{\prime}\right)} .
\end{aligned}
$$

Therefore we have

$$
\begin{aligned}
\left|e(X, X)-\frac{d}{n} x^{2}+\left(\frac{d x}{n}-\sum_{v \in X} l_{v}\right)\right| & \leq \frac{\left(\begin{array}{c}
x \\
x^{\prime}
\end{array}\right)}{\left(\begin{array}{c}
x-2 \\
x^{\prime}-1
\end{array}\right)} \frac{\theta}{n} \sqrt{x^{\prime}\left(n-x^{\prime}\right)\left(x-x^{\prime}\right)\left(n-x+x^{\prime}\right)} \\
& \leq \frac{2 \theta}{n} x(n-x / 2)
\end{aligned}
$$

as desired.

Corollary 4. Suppose that a graph $G$ with $n$ vertices has average degree $d$ and the eigenvalues $\sigma_{i}$ of the combinatorial Laplacian satisfy $\left|d-\sigma_{i}\right| \leq \theta$ for $i \neq 0$. Then for a subset $X$ of vertices in $G$, the number $e(X)$ of edges with both endpoints in $X$ satisfy

$$
\left|2 e(X)-\frac{d|X|(|X|-1)}{n}\right| \leq \frac{2 \theta}{n}|X|(n-|X| / 2) .
$$

COROLlary 5. Suppose that a graph $G$ with $n$ vertices has average degree $d$ and the eigenvalues $\sigma_{i}$ of the combinatorial Laplacian satisfy $\left|d-\sigma_{i}\right| \leq \theta$ for $i \neq 0$. Then an independent subset in $G$ can have no more than $\theta n / d+1$ vertices. 
It remains to prove Theorem 4.

Proof of Theorem 4: Let $\varphi_{i}, i=0, \ldots, n-1$, denote orthonormal eigenvectors of the combinatorial Laplacian. The eigenvalue associated with $\varphi_{i}$ is $\sigma_{i}$. Then for $i=0, \varphi_{0}=\mathbf{1} / \sqrt{n}$ where $\mathbf{1}$ is the all 1's vector. Suppose we write

$$
\begin{aligned}
\psi_{X} & =\sum_{i=0}^{n-1} a_{i} \varphi_{i} \\
\psi_{Y} & =\sum_{i=0}^{n-1} b_{i} \varphi_{i} .
\end{aligned}
$$

Here, let $I$ denote the identity matrix. We consider

$$
\begin{aligned}
e(X, Y)+d|X \cap Y| & =\left\langle\psi_{X},(A+d I) \psi_{Y}\right\rangle \\
& =\left\langle\psi_{X},(D-L) \psi_{Y}\right\rangle+d\left\langle\psi_{X}, \psi_{Y}\right\rangle \\
& =\operatorname{vol}(X \cap Y)-\sum_{i \neq 0} \sigma_{i} a_{i} b_{i}+d \sum_{i} a_{i} b_{i} \\
& =\operatorname{vol}(X \cap Y)-\sum_{i \neq 0}\left(d-\sigma_{i}\right) a_{i} b_{i}+d a_{0} b_{0} .
\end{aligned}
$$

We note that

$$
\begin{aligned}
& a_{0}=\left\langle\psi_{X}, \phi_{0}\right\rangle=\frac{|X|}{\sqrt{n}}, \\
& b_{0}=\left\langle\psi_{Y}, \phi_{0}\right\rangle=\frac{|Y|}{\sqrt{n}} .
\end{aligned}
$$

Thus, we have

$$
e(X, Y)+d|X \cap Y|=\operatorname{vol}(X \cap Y)-\sum_{i \neq 0}\left(d-\sigma_{i}\right) a_{i} b_{i}+\frac{d}{n}|X||Y| .
$$

Hence,

$$
\begin{aligned}
& \left|e(X, Y)-\frac{d}{n}\right| X|| Y|+d| X \cap Y|-\operatorname{vol}(X \cap Y)| \\
\leq & \sum_{i \neq 0}\left(d-\sigma_{i}\right) a_{i} b_{i} \\
\leq & \theta \sum_{i \neq 0}\left|a_{i} b_{i}\right| \\
\leq & \theta \sqrt{\sum_{i \neq 0} a_{i}^{2} \sum_{j \neq 0} b_{j}^{2}} \\
\leq & \theta \sqrt{\left(\left\|\psi_{X}\right\|^{2}-a_{0}^{2}\right)\left(\left\|\psi_{Y}\right\|^{2}-b_{0}^{2}\right)} \\
\leq & \theta \frac{\sqrt{(|X|(n-|X|)|Y|(n-|Y|)}}{n}
\end{aligned}
$$

as claimed.

In the preceding proof, if we use $d^{\prime}=\left(\sigma_{1}+\sigma_{n-1}\right) / 2$ in place of $d$, then we have the following: 
THEOREM 7. Suppose that in a graph $G$ with $n$ vertices, the combinatorial Laplacian has eigenvalues $0=\sigma_{0} \leq \sigma_{1} \ldots \leq \sigma_{n-1}$. Then for any two subsets $X$ and $Y$ of vertices in $G$, the number $e(X, Y)$ of edges with one endpoint in $X$ and the other in $Y$ satisfies

$$
\begin{aligned}
& \left|e(X, Y)-\frac{d^{\prime}}{n}\right| X|| Y\left|+d^{\prime}\right| X \cap Y|-\operatorname{vol}(X \cap Y)| \\
\leq & \frac{\left(\sigma_{n-1}-\sigma_{1}\right)}{2} \sqrt{|X|(n-|X|)|Y|(n-|Y|)}
\end{aligned}
$$

where $d^{\prime}=\left(\sigma_{n-1}+\sigma_{1}\right) / 2$.

\section{Cheeger inequalities}

In a graph $G=(V, E)$ and a subset $X$ of vertices, we define several versions of neighborhoods and boundaries.

- The neighborhood $N(X)$ is defined to be

$$
N(X)=\{v: v \in X \text { or } v \sim u \in X\}
$$

where $v \sim u$ means $\{u, v\}$ is an edge.

- The exact neighborhood $\Gamma(X)$ is just

$$
\Gamma(X)=\{v: v \sim u \in X\} .
$$

- The vertex boundary $\delta(X)$ of a subset $X$ of vertices is $\delta(X)=\{v \notin X$ : $v \sim$ $u \in X\}$ In general, for an integer $k \geq 1$, the $k$-neighborhood $N_{k}(X)$ of $X$ is defined to be $N_{k}(X)=N\left(N_{k-1}(X)\right)$ and $N_{0}(X)=X$. The $k$-boundary $\delta_{k}(X)$ is just $N_{k}(X) \backslash N_{k-1}(X)$.

- The edge boundary $\partial(X)$ is $\partial(X)=e(X, \bar{X})$. where $\bar{X}$ is the complement of $X$.

5.1. Isoperimetric inequalities for edge boundaries. The edge boundary is closely related to the discrete Cheeger's constant, which is defined as follows (see $[15,13])$.

$$
h_{G}=\inf _{X} \frac{|\partial(X)|}{\min \{\operatorname{vol}(X), \operatorname{vol}(\bar{X})\}} .
$$

The eigenvalues of the normalized Laplacian are related to Cheeger's constant by the discrete Cheeger inequality:

Clearly, this implies

$$
2 h_{G} \geq \lambda_{1} \geq \frac{h_{G}^{2}}{2}
$$

$$
\frac{|\partial(X)|}{\operatorname{vol}(X)} \geq h_{G} \geq \frac{\lambda_{1}}{2}
$$

if $\operatorname{vol}(X) \leq \operatorname{vol}(\bar{X})$. For subsets with given cardinality, a slightly stronger lower bound is as follows:

Lemma 1. For a graph $G$ and a subset of vertices $X$ with $\operatorname{vol}(X) \leq \operatorname{vol}(G) / 2$, we have

$$
\frac{|\partial(X)|}{\operatorname{vol}(X)} \geq \lambda_{1}\left(1-\frac{\operatorname{vol}(X)}{\operatorname{vol}(G)}\right) \geq \frac{\lambda_{1}}{2}
$$

where $\lambda_{1}$ is the least non-trivial eigenvalue of the normalized Laplacian of $G$.

The proof follows from the definition of $\lambda_{1}$ and can be found in [13]. Along the same line, the following holds for the combinatorial Laplacian. 
Lemma 2. For a graph $G$ on $n$ vertices and a subset of vertices $X$ with $|X| \leq$ $n / 2$, we have

$$
\frac{|\partial(X)|}{|X|} \geq \sigma_{1}\left(1-\frac{|X|}{n}\right) \geq \frac{\sigma_{1}}{2}
$$

where $\sigma_{1}$ is the least non-trivial eigenvalue of the combinatorial Laplacian of $G$.

We note that the Cheeger inequality used in Lemma 1 can be slightly improved (see $[\mathbf{1 3}])$.

$$
2 h_{G} \geq \lambda_{1}>1-\sqrt{1-h_{G}^{2}} .
$$

Another characterization of the Cheeger constant $h_{G}$ of a graph $G$ can be described as follows (see $[\mathbf{1 3}])$ :

$$
h_{G}=\inf _{f} \sup _{c \in \mathbb{R}} \frac{\sum_{x \sim y}|f(x)-f(y)|}{\sum_{x \in V}|f(x)-c| d_{x}}
$$

where $f$ ranges over all functions $f: V \rightarrow \mathbb{R}$ which are not identically zero.

A variation of (3) seems to be particularly useful, e.g., for deriving isoperimetric relationships between graphs and their Cartesian products [24].

$$
h_{G} \geq \inf _{f \neq 0} \frac{\sum_{x \sim y}|f(x)-f(y)|}{\sum_{x \in V}|f(x)| d_{x}} \geq \frac{1}{2} h_{G}
$$

where $f: V(G) \rightarrow \mathbb{R}$ satisfies $\sum_{x \in V} f(x) d_{x}=0$.

5.2. Isoperimetric inequalities for vertex boundaries. We will prove the following basic isoperimetric inequality:

THEOREM 8. Suppose that a graph $G$ with $n$ vertices has average degree $d$ and the eigenvalues $\sigma_{i}$ of the combinatorial Laplacian satisfy $\left|d-\sigma_{i}\right| \leq \theta$ for $i \neq 0$. Then for any nonempty subset $X$, the boundary $\delta(X)$ of $X$ satisfies

$$
\frac{|\delta(X)|}{|X|} \geq \frac{d^{2}-\theta^{2}}{\theta^{2}+d^{2}|X| /(n-|X|)}
$$

Proof. We use Theorem 4 and choose $Y=V(G) \backslash X \backslash \delta(X)$. Since $e(X, Y)=\emptyset$, we have

$$
\frac{d}{n}|X||Y| \leq \theta \frac{\sqrt{|X|(n-|X|)|Y|(n-|Y|)}}{n} .
$$

Thus,

$$
d^{2}|X||Y| \leq \theta^{2}(|X|+|\delta(X)|)(|Y|+|\delta(X)|)
$$

This implies

$$
\begin{aligned}
\frac{d^{2}-\theta^{2}}{\theta^{2}}|X||Y| & \leq|\delta(X)|(|X|+|Y|+|\delta(X)|) \\
& \leq|\delta(X)| n .
\end{aligned}
$$


Therefore,

$$
\frac{d^{2}-\theta^{2}}{\theta^{2} n}|X|(n-|X|-|\delta(X)|) \leq|\delta(X)|
$$

which is equivalent to

$$
\frac{d^{2}-\theta^{2}}{\theta^{2} n}|X|(n-|X|) \leq|\delta(X)|\left(1+\frac{d^{2}-\theta^{2}}{\theta^{2} n}|X|\right) .
$$

Finally, we have

$$
\begin{aligned}
\frac{|\delta(X)|}{|X|} & \geq \frac{\left(d^{2}-\theta^{2}\right)(n-|X|)}{\theta^{2} n+\left(d^{2}-\theta^{2}\right)|X|} \\
& =\frac{\left(d^{2}-\theta^{2}\right)(n-|X|)}{\theta^{2}(n-|X|)+d^{2}|X|} \\
& =\frac{d^{2}-\theta^{2}}{\theta^{2}+d^{2}|X| /(n-|X|)}
\end{aligned}
$$

as claimed.

As an immediate consequence of Theorem 8, we have the following three corollaries:

Corollary 6. Suppose that a graph $G$ with $n$ vertices has average degree $d$ and the eigenvalues $\sigma_{i}$ of the combinatorial Laplacian satisfy $\left|d-\sigma_{i}\right| \leq \theta$ for $i \neq 0$. For any subset $X$ with $|X| \ll n \theta^{2} / d^{2}$, the neighborhood $N(X)=X \cup \delta(X)$ of $X$ satisfies

$$
\frac{|N(X)|}{|X|} \geq(1+o(1)) \frac{d^{2}}{\theta^{2}} .
$$

Corollary 7. Suppose that a graph $G$ with $n$ vertices has average degree $d$ and the eigenvalues $\sigma_{i}$ of the combinatorial Laplacian satisfy $\left|d-\sigma_{i}\right| \leq \theta$ for $i \neq 0$. For any subset $X$ with $|X| \ll n \theta^{2 t} / d^{2 t}$, the $t$-neighborhood $N_{t}(X)=N\left(N_{t-1}(X)\right)$ of $X$ satisfies

$$
\frac{\left|N_{t}(X)\right|}{|X|} \geq(1+o(1)) \frac{d^{2 t}}{\theta^{2 t}}
$$

Corollary 8. Suppose that a graph $G$ with $n$ vertices has average degree $d$ and the eigenvalues $\sigma_{i}$ of the combinatorial Laplacian satisfy $\left|d-\sigma_{i}\right| \leq \theta$ for $i \neq 0$. For any vertex $v$, we have

$$
\delta_{t}(v) \geq \frac{(d-\theta)\left(d^{2 t-2}-\theta^{2 t-2}\right)}{\theta^{2 t-2}}\left(1-\frac{d^{2}}{n\left(d^{2}-\theta^{2}\right)}\left(\frac{d^{2}}{\theta^{2}}\right)^{t-1}\right) .
$$

In particular, for $\theta=o(d)$, we have

$$
\delta_{t}(v) \geq(1+o(1)) \frac{d^{2 t-1}}{\theta^{2 t-2}}\left(1-\frac{1}{n}\left(\frac{d^{2}}{\theta^{2}}\right)^{t-1}\right) .
$$

Proof. For the case of $t=1$, inequality (4) follows from Corollary 3. For $t>1$, inequality (4) is proved by using Corollary 6 and 7 .

Remark 1: The isoperimetric inequality for regular graphs by Tanner [61] and Alon [4] is a special case of Theorem 8 . 


\section{Diameter-eigenvalue inequalities}

In a graph $G$, the distance between two vertices $u$ and $v$, denoted by $d(u, v)$, is defined to be the length of a shortest path joining $u$ and $v$ in $G$. The diameter of $G$, denoted by $D(G)$, is the maximum distance over all pairs of vertices in G. Although the diameter is a combinatorial invariant, it is closely related to eigenvalues. In [14], the following relation between the diameter and eigenvalues holds for regular graphs (except for complete graphs).

$$
D(G) \leq\left\lceil\frac{\log (n-1)}{\log (1 /(1-\lambda))}\right\rceil .
$$

Here, $\lambda$ is $\lambda=\lambda_{1}$ if $1-\lambda_{1} \geq \lambda_{n-1}-1$.

6.1. Eigenvalues and diameters. Inequality (5) can be generalized to all graphs by using the combinatorial Laplacian [19].

TheOrem 9. Suppose a graph $G$ on $n$ vertices has eigenvalues $0 \leq \sigma_{1} \leq \ldots \leq$ $\sigma_{n-1}$. The diameter of $G$ satisfies

$$
D(G) \leq\left\lceil\frac{\log (n-1)}{\log \frac{\sigma_{n-1}+\sigma_{1}}{\sigma_{n-1}-\sigma_{1}}}\right\rceil .
$$

We note that for some graphs the above bound gives a pretty good upper bound for the diameter. For example, for $k$-regular Ramanujan graphs, we have $1-\lambda_{1}=\lambda_{n-1}-1=1 /(2 \sqrt{k-1})$, so we get $D \leq \log (n-1) /(2 \log (k-1))$, which is within a factor of 2 of the best possible bound.

The bound in (5) can be further improved by using the Chebyshev polynomial of degree $t$. We can then replace the logarithmic function by $\cosh ^{-1}$ (see [19]) :

$$
D(G) \leq\left\lceil\frac{\cosh ^{-1}(n-1)}{\cosh ^{-1} \frac{\sigma_{n-1}+\sigma_{1}}{\sigma_{n-1}-\sigma_{1}}}\right\rceil .
$$

6.2. Distances between two subsets. Instead of considering distances between two vertices, we can relate the eigenvalue $\lambda_{1}$ to distances between two subsets of vertices. For two subsets $X, Y$ of vertices in $G$, the distance between $X$ and $Y$, denoted by $d(X, Y)$, is the minimum distance between a vertex in $X$ and a vertex in $Y$; i.e., $d(X, Y)=\min \{d(x, y): x \in X, y \in Y\}$. In [31], the distance between two sets can be related to eigenvalues as follows:

TheOrem 10. Suppose $G$ is not a complete graph. For $X, Y \subset V(G)$,

$$
d(X, Y) \leq\left\lceil\frac{\log \sqrt{\frac{\operatorname{vol} \bar{X} \operatorname{vol} \bar{Y}}{\operatorname{vol} X \operatorname{vol} Y}}}{\log \frac{\lambda_{n-1}+\lambda_{1}}{\lambda_{n-1}-\lambda_{1}}}\right\rceil .
$$

6.3. Higher order eigenvalues and distances among many subsets. For any $k>1$, we can relate the eigenvalue $\lambda_{k}$ to distances among $k+1$ distinct subsets of vertices $[\mathbf{3 1}]$. 
Theorem 11. Suppose $G$ is not a complete graph. For $X_{i} \subset V(G), i=$ $0,1, \cdots, k$, we have

$$
\min _{i \neq j} d\left(X_{i}, X_{j}\right) \leq \max _{i \neq j}\left\lceil\frac{\log \sqrt{\frac{\operatorname{vol} \bar{X}_{i} \operatorname{vol} \bar{X}_{j}}{\operatorname{vol} X_{i} \operatorname{vol} X_{j}}}}{\log \frac{1}{1-\lambda_{k}}}\right\rceil
$$

if $1-\lambda_{k} \geq \lambda_{n-1}-1$.

We note that the condition $1-\lambda_{k} \geq \lambda_{n-1}-1$ can be eliminated by modifying $\lambda_{k}$ : For $X_{i} \subset V(G), i=0,1, \cdots, k$, we have

$$
\min _{i \neq j} d\left(X_{i}, X_{j}\right) \leq \max _{i \neq j}\left\lceil\frac{\log \sqrt{\frac{\operatorname{vol} \bar{X}_{i} \operatorname{vol} \bar{X}_{j}}{\operatorname{vol} X_{i} \operatorname{vol} X_{j}}}}{\log \frac{\lambda_{n-1}+\lambda_{k}}{\lambda_{n-1}-\lambda_{k}}}\right\rceil
$$

if $\lambda_{k} \neq \lambda_{n-1}$.

Another useful generalization is the following: For $X_{i} \subset V(G), i=0,1, \cdots, k$, we have

$$
\min _{i \neq j} d\left(X_{i}, X_{j}\right) \leq \min _{0 \leq j<k} \max _{i \neq j}\left\lceil\frac{\log \sqrt{\frac{\operatorname{vol} \bar{X}_{i} \operatorname{vol} \bar{X}_{j}}{\operatorname{vol} X_{i} \operatorname{vol} X_{j}}}}{\log \frac{\lambda_{n-j-1}+\lambda_{k-j}}{\lambda_{n-j-1}-\lambda_{k-j}}}\right\rceil
$$

where $j$ satisfies $\lambda_{k-j} \neq \lambda_{n-j-1}$.

6.4. Eigenvalue upper bounds for manifolds. The above discrete methods can be used to derive new eigenvalue upper bounds for compact smooth Riemannian manifolds $[\mathbf{2 1}, \mathbf{2 2}]$. Let $M$ be a complete Riemannian manifold with finite volume and let $\mathcal{L}$ be the self-adjoint operator $-\Delta$, where $\Delta$ is the Laplace operator associated with the Riemannian metric on $M$. Or, we could consider a compact Riemannian manifold $M$ with boundary and let $\mathcal{L}$ be a self-adjoint operator $-\Delta$ subject to the Neumann or Dirichlet boundary conditions.

The operator $\mathcal{L}=-\Delta$ is self-adjoint and has a discrete spectrum in $L^{2}(M, \mu)$, where $\mu$ denotes the Riemannian measure. Let the eigenvalues be denoted by $0=\lambda_{0}<\lambda_{1} \leq \lambda_{2} \leq \cdots$. Let $\operatorname{dist}(x, y)$ be a distance function on $M \times M$ which is Lipschitz and satisfies

$$
|\nabla \operatorname{dist}(x, y)| \leq 1
$$

for all $x, y \in M$. For example, $\operatorname{dist}(x, y)$ may be taken to be the geodesic distance, but we don't necessarily assume this is the case.

Using very similar methods as in the discrete case, it can be shown that (see $[21])$ :

For two arbitrary measurable disjoint sets $X$ and $Y$ on $M$, we have

$$
\lambda_{1} \leq \frac{1}{\operatorname{dist}(X, Y)^{2}}\left(1+\log \frac{(\mu M)^{2}}{\mu X \mu Y}\right)^{2} .
$$

Moreover, if we have $k+1$ disjoint subsets $X_{0}, X_{1}, \cdots, X_{k}$ such that the distance between any pair of them is greater than or equal to $D>0$, then we have for any $k \geq 1$,

$$
\lambda_{k} \leq \frac{1}{D^{2}}\left(1+\sup _{i \neq j} \log \frac{(\mu M)^{2}}{\mu X_{i} \mu X_{i}}\right)^{2} .
$$

Although differential geometry and spectral graph theory have a great deal in common, there is no question that significant differences exist. Obviously, a 
graph is not "differentiable", and many geometrical techniques involving high-order derivatives could be very difficult, if not impossible, to utilize for graphs. There are substantial obstacles for deriving the discrete analogs of many of the known results in the continuous case. Nevertheless, the above result is an example of mutual fertilization that shed insight to both the continuous and discrete cases.

\section{Sobolev constants and Sobolev inequalities}

The characterization of the Cheeger constant is basically the Rayleigh quotient using the $L_{1}$-norm both in the numerator and denominator. In general, we can consider the so-called Sobolev constant for all $p, q>0$ :

$$
\begin{aligned}
s_{p, q} & =\inf _{f} \frac{\sum_{u \sim v}|f(u)-f(v)|^{p}}{\sum_{v}|f(v)|^{q} d_{v}} \\
& =\inf _{f} \frac{\int|\nabla f|^{p}}{\int|f|^{q}}
\end{aligned}
$$

where $f$ ranges over functions satisfying

$$
\sum_{x}|f(x)-c|^{q} d_{x} \geq \sum_{x}|f(x)|^{q} d_{x}
$$

for any $c$, or, equivalently,

$$
\int|f-c|^{q} \geq \int|f|^{q}
$$

The eigenvalue $\lambda_{1}$ corresponds to the case of $p=q=2$, while the Cheeger constant is associated with the case of $p=q=1$.

There are many common concepts that provide connections and interactions between spectral graph theory and Riemannian geometry. For example, the Sobolev inequalities for graphs can be proved almost entirely by classical techniques which can be traced back to Nash [64]. We will describe Sobolev inequalities which hold for all general graphs. However, such inequalities depend on a graph invariant, the so-called isoperimetric dimension.

We say that a graph $G$ has isoperimetric dimension $\delta$ with an isoperimetric constant $c_{\delta}$ if for all subsets $X$ of $V(G)$, the number of edges between $X$ and the complement $\bar{X}$ of $X$, denoted by $e(X, \bar{X})$, satisfies

$$
e(X, \bar{X}) \geq c_{\delta}(\operatorname{vol} X)^{\frac{\delta-1}{\delta}}
$$

where vol $X \leq \operatorname{vol} \bar{X}$ and $c_{\delta}$ is a constant depending only on $\delta$. Let $f$ denote an arbitrary function $f: V(G) \rightarrow \mathbb{R}$. The following Sobolev inequalities hold [25].

(i) For $\delta>1, \sum_{u \sim v}|f(u)-f(v)| \geq c_{1} \min _{m}\left(\sum_{v}|f(v)-m|^{\frac{\delta}{\delta-1}} d_{v}\right)^{\frac{\delta-1}{\delta}}$

(ii) For $\delta>2,\left(\sum_{u \sim v}|f(u)-f(v)|^{2}\right)^{\frac{1}{2}} \geq c_{2} \min _{m}\left(\sum_{v} \mid(f(v)-m)^{\alpha} d_{v}\right)^{\frac{1}{\alpha}}$ where $\alpha=\frac{2 \delta}{\delta-2}$ and $c_{1}, c_{2}$ are constants depending only on $c_{\delta}$. 
The above two inequalities can be used to derive the following eigenvalue inequalities for a graph $G$ (see $[\mathbf{2 5}])$ :

$$
\begin{aligned}
\sum_{i>0} e^{-\lambda_{i} t} & \leq c \frac{\operatorname{vol}(G)}{t^{\frac{\delta}{2}}} \\
\lambda_{k} & \geq c^{\prime}\left(\frac{k}{\operatorname{vol}(G)}\right)^{\frac{2}{\delta}}
\end{aligned}
$$

for suitable constants $c$ and $c^{\prime}$ which depend only on $\delta$ and $c_{\delta}$. In a way, a graph can be viewed as a discretization of a Riemannian manifold in $\mathbb{R}^{n}$ where $n$ is roughly equal to $\delta$. The eigenvalue bounds above are analogs of the Polyá conjecture for Dirichlet eigenvalues of a regular domain $M$.

$$
\lambda_{k} \geq \frac{2 \pi}{w_{n}}\left(\frac{k}{\operatorname{vol} M}\right)^{\frac{2}{n}}
$$

where $w_{n}$ is the volume of the unit disc in $\mathbb{R}^{n}$.

In a later paper $[\mathbf{2 3}]$, the condition in $(7)$ is further relaxed. It was shown that if in a graph $G=(V, E)$, any subset $X \subseteq V$ satisfies

$$
e(X, \bar{X}) \geq c(\operatorname{vol}(X))^{(\delta-1) / \delta}
$$

for $\operatorname{vol}(X) \leq c_{1}$, then the Dirichlet eigenvalue $\lambda_{k}(S)$ for the induced subgraph $S$ satisfies

$$
\lambda_{k}(S) \geq c^{\prime}\left(\frac{k}{\operatorname{vol}(S)}\right)^{2 / \delta}
$$

where $c^{\prime}$ depends on $\delta$ and $c$, provided $|S| \geq k \geq \frac{(2+\delta) e}{c_{1}} \operatorname{vol}(S)$.

An interesting question is to deduce the isoperimetric dimension or inequalities such as (7) from an arbitrarily given graph. In [23], we examine certain sufficient conditions on graph distance functions and their modifications for deriving (7).

\section{Harnack inequalities}

A crucial part of spectral graph theory concerns understanding the behavior of eigenfunctions. Intuitively, an eigenfunction maps the vertices of a graph to the real line in such a way that edges serve as "elastic bands" with the effect of pulling adjacent vertices closer together. To be specific, let $f$ denote an eigenfunction with eigenvalue $\lambda$ in a graph $G$ (or for an induced subgraph $S$ with nonempty boundary). Locally, at each vertex, the eigenfunction stretches the incident edges in a balanced way. That is, for each vertex $x, f$ satisfies

$$
\sum_{\substack{y \\ \sim x}}(f(x)-f(y))=\lambda f(x) d_{x} .
$$

Globally, we would like to have some notion that adjacent vertices are close to one another. In spectral geometry, Harnack inequalities are exactly the tools for capturing the essence of eigenfunctions. There are many different versions of Harnack inequalities (involving constants depending on the dimension of the manifold, for example). We consider the following inequality for graphs.

\section{A Harnack inequality:}

For every vertex $x$ in a graph $G$ and some absolute constant $c$, any eigenfunction 
$f$ with eigenvalue $\lambda$ satisfies

$$
\frac{1}{d_{x}} \sum_{\substack{y \\ y \sim x}}(f(x)-f(y))^{2} \leq c \lambda \max _{z} f^{2}(z) .
$$

However, the above inequality does not hold for all graphs in general. An easy counterexample is the graph formed by joining two complete graphs of the same size by a single edge. Nevertheless, we can establish a Harnack inequality for certain homogeneous graphs and their "strongly convex subgraphs".

A homogeneous graph is a graph $\Gamma$ together with a group $H$ acting on the vertices satisfying:

1. For any $g \in H, u \sim v$ if and only if $g u \sim g v$.

2. For any $u, v \in V(\Gamma)$ there exists $g \in H$ such that $g u=v$.

In other words, $\Gamma$ is vertex transitive under the action of $H$, and the vertices of $\Gamma$ can be labeled by cosets $H / I$ where $I=\{g: g v=v\}$ for a fixed $v$. Also, there is an edge-generating set $K \subset H$ such that for all vertices $v \in V(\Gamma)$ and $g \in K$, we have $\{v, g v\} \in E(\Gamma)$. A homogeneous graph is said to be invariant if $K$ is invariant as a set under conjugation by elements of $K$, i.e., for all $a \in K, a K a^{-1}=K$.

Let $f$ denote an eigenfunction in an invariant homogeneous graph with edgegenerating set $K$ consisting of $k$ generators. Then it can be shown [26] that

$$
\frac{1}{k} \sum_{a \in K}(f(x)-f(a x))^{2} \leq 8 \lambda \sup _{y} f^{2}(y) .
$$

An induced subgraph $S$ of a graph $\Gamma$ is said to be strongly convex if for all pairs of vertices $u$ and $v$ in $S$, all shortest paths joining $u$ and $v$ in $\Gamma$ are contained in $S$. The main theorem in [26] asserts that the following Harnack inequality holds.

Suppose $S$ is a strongly convex subgraph in an abelian homogeneous graph with edge-generating set $K$ consisting of $k$ generators. Let $f$ denote an eigenfunction of $S$ associated with the Neumann or Dirichlet eigenvalue $\lambda$. Then for all $x \in S$, $x \sim y$,

$$
|f(x)-f(y)|^{2} \leq 8 k \lambda \sup _{z \in S} f^{2}(z) .
$$

(The detailed definition of Neumann or Dirichlet eigenvalues of an induced subgraph will be given in the next section.)

A direct consequence of the Harnack inequalities is the following lower bound for the Neumann or Dirichlet eigenvalue $\lambda_{1}$ of $S$ :

$$
\lambda_{1} \geq \frac{1}{8 k D^{2}}
$$

where $k$ is the maximum degree and $D$ is the diameter of $S$. Such eigenvalue bounds are particularly useful for deriving polynomial approximation algorithms when enumeration problems of combinatorial structures can be represented as random walk problems on "convex" subgraphs of appropriate homogeneous graphs. However, the condition of being a strongly convex subgraph poses quite severe constraints, which will be relaxed in the next section.

\section{Heat kernel eigenvalue inequalities}

In a graph $G$, for a subset $S$ of the vertex set $V=V(G)$, the induced subgraph determined by $S$ has edge set consisting of all edges of $G$ with both endpoints in $S$. Although an induced subgraph can also be viewed as a graph in its own right, 
it is natural to consider an induced subgraph $S$ as having a boundary. For an induced subgraph $S$ with non-empty boundary, there are, in general, two kinds of eigenvalues - the Neumann eigenvalues and the Dirichlet eigenvalues - subject to different boundary conditions.

For the Neumann eigenvalues, the Laplacian $\mathcal{L}$ acts on functions $f: S \cup \delta S \rightarrow \mathbb{R}$ with the Neumann boundary condition, i.e., for every $x \in \delta S, \sum_{y \in S, y \sim x}(f(x)-$ $f(y))=0$.

For the Dirichlet eigenvalues, the Laplacian $\mathcal{L}$ acts on functions with the Dirichlet boundary condition. In other words, we consider the space of functions $\{f: V \rightarrow \mathbb{R}\}$ which satisfy the Dirichlet condition $f(x)=0$ for any vertex $x$ in the vertex boundary $\delta S$ of $S$.

The Neumann boundary condition corresponds to the Neumann boundary condition for Riemannian manifolds: $\frac{\partial f(x)}{\partial \nu}=0$ for $x$ on the boundary where $\nu$ is the normal direction orthogonal to the tangent hyperplane at $x$. Neumann eigenvalues are closely associated with random walk problems, whereas the Dirichlet eigenvalues are related to many boundary-value problems (Details can be found in [13]). In the remainder of this section, we will focus on Neumann eigenvalues and the heat kernel techniques to obtain eigenvalue lower bounds. In the literature, there are many general formulations for discrete heat kernel in connection with the continuous heat kernels $[\mathbf{3 4}, \mathbf{4 0}]$. Here we define a natural heat kernel for general graphs.

Let $\phi_{i}$ denote the eigenfunction for the Laplacian corresponding to eigenvalue $\lambda_{i}$. We now define the heat kernel of $S$ as a $n \times n$ matrix

$$
\begin{aligned}
H_{t} & =\sum e^{\lambda_{i} t} P_{i} \\
& =e^{-t \mathcal{L}} \\
& =I-t \mathcal{L}+\frac{t^{2}}{2} \mathcal{L}+\cdots
\end{aligned}
$$

where $\mathcal{L}=\sum \lambda_{i} P_{i}$ is the decomposition of the Laplacian $\mathcal{L}$ into projections on its eigenspaces. In particular, we have $H_{0}=I$, and for $F(x, t)=\sum_{y \in S \cup \delta S} H_{t}(x, y) f(y)=$ $\left(H_{t} f\right)(x)$ with $F(x, 0)=f(x), F$ satisfies the heat equation

$$
\frac{\partial F}{\partial t}=-\mathcal{L} F
$$

By using the heat kernel, the following eigenvalue inequality can be derived, for all $t>0$ :

$$
\lambda_{S} \geq \frac{1}{2 t} \sum_{x \in S} \inf _{y \in S} H_{t}(x, y) \frac{\sqrt{d_{x}}}{\sqrt{d_{y}}} .
$$

One way to use the above theorem is to bound the heat kernel of a graph by the (continuous) heat kernel of Riemannian manifolds. We say $\Gamma$ is a lattice graph if $\Gamma$ is embedded into a $d$-dimensional Riemannian manifold $\mathcal{M}$ with a metric $\mu$ such that $\epsilon=\mu(x, g x)=\mu\left(y, g^{\prime} y\right)$ for all $g, g^{\prime} \in K$ and $x, y \in V(\Gamma)$. An induced subgraph on a subset $S$ of a lattice graph $\Gamma$ is said to be convex if there is a submanifold $M \subset \mathcal{M}$ with a convex boundary $\partial M \neq \emptyset$ such that $S$ consists of all vertices of $\Gamma$ in the interior of $M$. Furthermore, we require that for any vertex $x$, the Vorono $i$ region $R_{x}=\{y: \mu(y, x)<\mu(y, z)$ for all $z \in \Gamma \cap \mathcal{M}\}$ is contained in $M$. 
One additional condition is needed here. Basically, $\epsilon$ has to be "small" enough so that the count of vertices in $S$ can be used to approximate the volume of the manifold $M$. Namely, let us define

$$
r=\frac{U|S|}{\operatorname{vol}(M)}
$$

where $U$ denotes the maximaum volume of a Vorono $i$ region. Then the main result in [28] states that the Neumann eigenvalue $\lambda_{1}$ of $S$ satisfies the following inequality:

$$
\lambda_{1} \geq \frac{c r \epsilon^{2}}{d^{2} D^{2}(M)}
$$

for some absolute constant $c$, which depends only on $\Gamma$, and $D(M)$ denotes the diameter of the manifold $M$. We note that $r$ in (9) can be bounded below by a constant if the diameter of $M$ measured in $L_{1}$ norm is at least $\epsilon d$. The applications on random walks in Section 14 will use the above eigenvalue inequality.

\section{Paths and cycles}

One of the major theorems in studying the paths of a graph is a result of Pósa [57] (see [54], Problem 10.20, for an elegant solution).

\section{Pósa's Theorem}

In a graph $H$ if every subset $X$ of vertices with $|X| \leq k$ satisfies

$$
|\delta(X)| \geq 2|X|-1
$$

then $H$ contains a path with $3 k-2$ vertices.

THEOREM 12. Suppose that a graph $G$ with $n$ vertices has average degree $d$ and the eigenvalues $\sigma_{i}$ of the combinatorial Laplacian satisfy $\left|d-\sigma_{i}\right| \leq \theta$ for $i \neq 0$. Then $G$ contains a path of at least

$$
\left(1-\frac{2 \theta^{2}}{d^{2}-\theta^{2}}\right) n-2 \text { vertices. }
$$

Proof. To deduce the existence of a path of $\frac{d^{2}-3 \theta^{2}}{d^{2}-\theta^{2}} n-2$ vertices, it suffices to show that we have $\delta(X) \geq 2|X|-1$ for any subset $X$ with cardinality at most

$$
|X| \leq \frac{d^{2}-3 \theta^{2}}{3\left(d^{2}-\theta^{2}\right)} n=x_{0}
$$

by using Pósa's Theorem. From Theorem 8 , we know that

$$
\frac{|\delta(X)|}{|X|} \geq \frac{d^{2}-\theta^{2}}{\theta^{2}+d^{2} x_{0} /\left(n-x_{0}\right)} .
$$

After substituting for $x_{0}$, we have

$$
\frac{|\delta(X)|}{|X|} \geq 2
$$

Theorem 12 is proved.

THEOREM 13. Suppose that a graph $G$ with $n$ vertices has average degree $d$ and the eigenvalues $\sigma_{i}$ of the combinatorial Laplacian satisfy $\left|d-\sigma_{i}\right| \leq \theta$ for $i \neq 0$. If $d^{2 k} \gg n \theta^{2 k-1}$, then $G$ contains a cycle of length $2 k+1$, if $n$ is sufficiently large. 
Proof. We consider $\delta_{i}(v)=\{u: d(u, v)=i\}$. In a paper [38] by Erdős et al., it was shown that if a graph $G$ contains no cycle of length $2 k+1$, then for any $1 \leq i \leq k$ the induced subgraph on $\delta_{i}(v)$ contains an independent set $S$ of size at least $\left|\delta_{i}(v)\right| /(2 k-1)$. From Theorem 6 , we have

$$
d|S|(|S|-1)<2 \theta|S|(n-|S| / 2) .
$$

This implies that

$$
|S| \leq \frac{2 \theta n}{d}
$$

(Here we use the fact that $\theta \geq \sqrt{d}$.) Hence, we have $\left|\delta_{i}(v)\right| /(2 k-1) \leq 2 \theta n / d$.

Since $\theta=o(d)$, by Corollary 8 , we have

$$
\left|\delta_{k}(v)\right| \geq(1+o(1)) \frac{d^{2 k-1}}{\theta^{2 k-2}}\left(1-\frac{1}{n}\left(\frac{d^{2}}{\theta^{2}}\right)^{t-1}\right) .
$$

Thus, we have

$$
\frac{2(2 k-1) \theta n}{d} \geq(1+o(1)) \frac{d^{2 k-1}}{\theta^{2 k-2}}\left(1-\frac{1}{n}\left(\frac{d^{2}}{\theta^{2}}\right)^{t-1}\right) .
$$

This implies that

$$
n \geq(1+o(1)) \frac{d^{2 k}}{2(2 k-1) \theta^{2 k-1}}
$$

which is a contradiction to the assumption that $n \ll d^{2 k} / \theta^{2 k-1}$.

In [45] Krivelevich and Sudakov showed that a $d$-regualar graph on $n$ vertices is Hamiltonian if the eigenvalues of the combinatorial Laplacian satisfy

$$
\left|d-\sigma_{i}\right| \leq c \frac{(\log \log n)^{2}}{\log n(\log \log \log n)}
$$

for $i \neq 0$ and for some constant $c$. The method is a modified version of Posa's technique developed by Komlós and Szemerédi [44] for examining Hamiltonian cycles in random graphs. By using the discrepancy inequalities and the isoperimetric inequalities in previous sections, the above result can be extended to general graphs as well.

\section{Universal graphs for trees of bounded degrees}

There is quite a literature on so-called "universal graph" that contain all trees on $n$ vertices or other families of graphs such as trees with bounded degree $[\mathbf{5}$, $\mathbf{8}, \mathbf{1 6}, \mathbf{2 0}, \mathbf{3 9}$ ]. One of the main avenues in the study of universal graphs is the connection with expanding properties of the graph. Friedman and Pippenger [39] proved the following beautiful result:

Theorem [39] Suppose that $H$ is a graph such that for every subset $X$ of vertices with $|X| \leq 2 n-2, X$ has exact neighborhood $\Gamma(X)=\{u: u \sim v \in X\}$ satisfying

$$
|\Gamma(X)| \geq(k+1)|X|
$$

Then $H$ contains every tree with $n$ vertices and maximum degree at most $k$.

Here we will prove the following slightly stronger result the proof of which will be given later. 
THEOREM 14. Suppose that $H$ is a graph such that for every subset $X$ of vertices with $|X| \leq 2 n-2$ has boundary $\delta(X)$ satisfying

$$
|\delta(X)| \geq k|X|
$$

then $H$ contains every tree with $n$ vertices and maximum degree at most $k$.

As an immediate consequence of Theorem 14, we have the following:

THEOREM 15. Suppose that a graph $G$ with $n$ vertices has average degree $d$ and the eigenvalues $\sigma_{i}$ of the combinatorial Laplacian satisfy $\left|d-\sigma_{i}\right| \leq \theta$ for $i \neq 0$. Then $G$ contains all trees with maximum degree $k$ and having at least

$$
\frac{d^{2}-(k+1) \theta^{2}}{2(k+1)\left(d^{2}-\theta^{2}\right)} n+1 \text { vertices. }
$$

Proof. To deduce the existence of a tree having at least

$$
t=\frac{d^{2}-(k+1) \theta^{2}}{2(k+1)\left(d^{2}-\theta^{2}\right)} n+1
$$

vertices and degree bounded above by $k$ it suffices to show that we have $\delta(X) \geq k|X|$ for any subset $X$ with size at most

$$
|X| \leq \frac{d^{2}-(k+1) \theta^{2}}{(k+1)\left(d^{2}-\theta^{2}\right)} n=z_{0} \leq 2 t-2
$$

by using Theorem 14 . From Theorem 8 , we know that

$$
\frac{|\delta(X)|}{|X|} \geq \frac{d^{2}-\theta^{2}}{\theta^{2}+d^{2} z_{0} /\left(n-z_{0}\right)} .
$$

After substituting for $z_{0}$, we have

$$
\frac{|\delta(X)|}{|X|} \geq k
$$

Theorem 15 is proved.

Corollary 9. Suppose that a graph $G$ with $n$ vertices has average degree $d$ and the eigenvalues $\sigma_{i}$ of the combinatorial Laplacian satisfy $\left|d-\sigma_{i}\right| \leq \theta$ for $i \neq 0$. If $\theta=o(d)$, then $G$ contains all trees with maximum degree $k$ and having at most

$$
(1+o(1)) \frac{n}{2(k+1)}
$$

vertices, if $n$ is sufficiently large.

It remains to prove Theorem 14 . The proof is quite similar to that in $[\mathbf{3 9}]$. For completeness, we sketch the proof here.

Proof of Theorem 14: Suppose that $T$ is a tree on $m$ vertices with maximum degree at most $k$. For an embedding $f: V(T) \rightarrow V(H)$, we define the excess $C(f, X)$ for $X \subseteq V(H)$ by

$$
\begin{aligned}
C(f, X) & :=A(f, X)-B(f, X) \\
\text { where } A(f, X) & =|X \cup \delta(X) \backslash f(V(T))| \\
\text { and } B(f, X) & =\sum_{x \in X}\left(k-\operatorname{deg}_{T} f^{-1}(x)\right)
\end{aligned}
$$

where $\operatorname{deg}_{T} f^{-1}(x)$ denotes the degree in $T$ of the vertex $u$ that is mapped to $x$ under $f$, or $\operatorname{deg}_{T} f^{-1}(x)$ is zero if no such $u$ exists. Since the maximum degree in 
the tree is $k, B(f, X) \geq 0$. An embedding $f$ is said to be good if for every $X \subseteq V(H)$ with $|X| \leq 2 m-2$, we have

$$
C(f, X) \geq 0 .
$$

Instead of proving Theorem 14, We will show a stronger statement:

(*) Suppose that $H$ is a graph such that for every subset $X$ of vertices with $|X| \leq 2 m-2$ has neighborhood $\Gamma(X)$ satisfying $|\delta(X)| \geq k|X|$.

Suppose that $S$ is a tree on $m$ vertices and maximum degree at most $k$. Furthermore, we assume that $S$ has a good embedding in $H$. Then a tree $T$ formed by adding any leaf to $T$ also has a good embedding.

We will prove $\left(^{*}\right)$ by induction on $m$. Clearly, $\left(^{*}\right)$ holds for the case of $S$ consisting of one single vertex. Suppose $\left(^{*}\right)$ holds for any tree on $m$ vertices having maximum degree $k$. We now consider a tree $T$ obtained from $S$ by adding a leaf $v$ and the incident edge $\{v, w\}$. Suppose $f$ is a good embedding for $S$. By the definition of being good, for each $g \in \delta(f(w)) \backslash f(V(S))$, we can extend $f$ to be an embedding $f_{g}$ for $T$ by mapping $v$ to $g$. We want to show that there is some good extended embedding $f_{g}$.

Suppose to the contrary that no extended embedding $f_{g}$ is good. For every $g \in \delta(f(w)) \backslash f(V(S))$, there is a subset $X_{g}$ of $V(H)$ such that $C\left(f_{g}, X_{g}\right)<0$. We have

Note that

$$
C\left(f_{g}, X_{q}\right)<0 \leq C\left(f, X_{g} \cap V(S)\right) .
$$

$$
C\left(f_{g}, X_{q}\right)=C\left(f, X_{q}\right)-\epsilon(g, X \cup \delta X)+\epsilon(f(w), X)+\epsilon(q, X),
$$

where $\epsilon(x, Y)$ is 1 if $x \in Y$ and 0 otherwise. Therefore, we have $g \notin X_{g}, f(w) \notin X_{g}$, $g \in \delta\left(X_{g}\right)$ and $C\left(f, X_{g}\right)=0$.

We say that $X$ is critical under $f$ if $C(f, X)=0$. We need the following facts, some of which have the same proofs as those in [39].

Fact 1: Suppose that $X$ is critical under $f$ and $|S| \leq m-1$. If $|X| \leq 2 m-2$, then $|X| \leq m-1$.

Proof: We have

$$
\begin{aligned}
0 & =C(f, X) \geq|X \cup \delta X|-|V(S)|-k|X| \\
& \geq(k+1)|X|-(m-1)-k|X| \\
& \geq|X|-(m-1) .
\end{aligned}
$$

Fact 2: The excess $C(f, \cdot)$ is submodular:

$$
C(f, X \cup Y)+C(f, X \cap Y) \leq C(f, X)+C(f, Y) .
$$

Fact 3: If $X, Y \subseteq V(H)$ are critical under $f$ so that $|X|,|Y| \leq m-1$, then $X \cup Y$ is critical under $f$ and $|X \cup Y| \leq m-1$.

Now we return to the proof of Theorem 14. For every $g \in \delta(f(w)) \backslash f(V(S))$, $X_{g}$ is critical under $f$ and $\left|X_{g}\right| \leq 2 m-2$. By Fact $1,\left|X_{g}\right| \leq m-1$. Furthermore, we consider $X^{*}=\cup_{g} X_{g}$, which by Fact 3 is critical under $f$ and $\left|X^{*}\right| \leq m-1$. Now consider $X^{\prime}=X^{*} \cup f(w)$ and $C\left(f, X^{\prime}\right)$. Since $f$ is a good embedding of $S$ and $\left|X^{\prime}\right| \leq m$, we have $C\left(f, X^{\prime}\right) \geq 0$.

For every $g \in \delta(f(w)) \backslash f(V(S))$, we have $g \in X^{*} \cup \delta\left(X^{*}\right)$ which implies $A\left(f, X^{\prime}\right)=A\left(f, X^{*}\right)$. However, $B\left(f, X^{\prime}\right)=B\left(f, X^{*}\right)+B(f, f(w))$ since $f(w) \notin$ $X_{g}$ for every $g$. Thus, $B\left(f, X^{\prime}\right)<B\left(f, X^{*}\right)$, and we have $C\left(f, X^{*}\right)<0$. This 
contradicts the assumption that $f$ is good. We conclude that there is an extended embedding $f_{g}$ which is good, and the proof of Theorem 14 is complete.

\section{Chromatic numbers and list chromatic numbers}

One of the basic topics in graph theory is graph coloring. For a graph $G$, the chromatic number $\chi(G)$ of $G$ is the least integer $m$ so that each vertex can be assigned one of the $k$ colors such that adjacent vertices have different colors. In the past ten years, there has been a great deal of work on extensions of chromatic numbers. In a graph $G$, suppose each vertex is associated with a list of $k$ colors. A proper coloring assigns to each vertex a color from its list so that no two adjacent vertices have the same color. The list chromatic number $\chi_{l}(G)$ is the least integer $k$ so that there is a proper coloring for any given color lists of length $k$. Clearly, we have

$$
\chi(G) \leq \chi_{l}(G) .
$$

We will use the following theorem by $\mathrm{Vu}[\mathbf{6 2}]$ :

Vu's Theorem : Suppose a graph $G$ has maximum degree $d$. If the neighborhood of each vertex in $V(G)$ contains at most $d^{2} / f$ edges, for some $d^{2}>f>1$, then

for some constant $K$.

$$
\chi_{l}(G) \leq K \frac{d}{\log f}
$$

THEOREM 16. Suppose that a graph $G$ with $n$ vertices has average degree $d$ and the eigenvalues $\sigma_{i}$ of the combinatorial Laplacian satisfy $\left|d-\sigma_{i}\right| \leq \theta$ for $i \neq 0$. Then the chromatic number $\chi(G)$ and the list chromatic number $\chi_{l}(G)$ satisfy

$$
\frac{d}{\theta}<\chi(G) \leq \chi_{l}(G) \leq O\left(\frac{d}{\log \left(\min \left\{\frac{n}{d}, \frac{d}{\theta}\right\}\right)}\right) .
$$

Proof. From Corollary 3 , we know that the maximum degree is at most $d+\theta$. Using Corollary 4 , any subgraph on $d+\theta$ vertices can have at most

$$
\frac{d(d+\theta)^{2}}{n}+2 \theta(d+\theta)
$$

edges. So, we can use Vu's Theorem by choosing $f$ satisfying

$$
\frac{1}{f}=\frac{d}{n}+\frac{2 \theta}{d}
$$

and therefore

$$
\begin{aligned}
\chi_{l}(G) & =O\left(\frac{d+\theta}{\log f}\right) \\
& =O\left(\frac{d}{\log \left(\min \left\{\frac{n}{d}, \frac{d}{\theta}\right\}\right)}\right) .
\end{aligned}
$$

In the other direction, we can establish a lower bound for $\chi(G)$ as well as $\chi_{l}(G)$, by using Corollary 5 again. Namely, an independent set in $G$ can have at most $\theta n / d$ vertices. Therefore

$$
\frac{d}{\theta} \leq \chi(G) \leq \chi_{l}(G)=O\left(\frac{d}{\log \left(\min \left\{\frac{n}{d}, \frac{d}{\theta}\right\}\right)}\right) .
$$




\section{Turán numbers}

A celebrated result in extremal graph theory is Turán's Theorem which states that a graph on $n$ vertices containing no $K_{t+1}$ can have at most $(1-1 / t+o(1))\left(\begin{array}{l}n \\ 2\end{array}\right)$ edges. Sudakov, Szabó and Vu [60] consider a generalization of Turán's Theorem. A graph $G$ with $e(G)$ edges is said to be $t$-Turán if any subgraph of $G$ containing no $K_{t+1}$ has at most $(1-1 / t+o(1)) e(G)$ edges. In [60], it is shown that a regular graph on $n$ vertices with degree $d$ is $t$-Turán if the second largest eigenvalue of its adjacency matrix $\lambda$ is sufficiently small. Their result can be extended to general graphs by using the isoperimetric and discrepancy inequalities [17].

TheOREM 17. Suppose a graph $G$ on $n$ vertices has eigenvalues of the normalized Laplacian

$0=\lambda_{0} \leq \lambda_{1} \leq \ldots \leq \lambda_{n-1}$ with $\bar{\lambda}=\max _{i \neq 0}\left|1-\lambda_{i}\right|$ satisfying

$$
\bar{\lambda}=o\left(\frac{1}{\operatorname{vol}_{-2 t+1}(G) \operatorname{vol}(G)^{t-1}}\right)
$$

where $\operatorname{vol}_{i}(S)=\sum_{x \in S} d_{x}^{i}$.

Then, $G$ is $t$-Turán for $t \geq 2$, i.e., any subgraph of $G$ containing no $K_{t+1}$ has at most $(1+1 / t+o(1)) e(G)$ edges where $e(G)$ is the number of edges in $G$.

\section{Random walks and contingency tables}

A walk is a sequence of vertices $w=\left(v_{0}, v_{1}, \cdots, v_{s}\right)$ such that $\left\{v_{i-1}, v_{i}\right\}$ is an edge. A Markov chain can be viewed as a random walk, defined by its transition probability matrix $P$, where the probability of moving from $u$ to $v$ is $P(u, v)$. Clearly, $P(u, v)>0$ only if $(u, v)$ is an edge. Also, $\sum_{v} P(u, v)=1$. For a weighted graph with edge weights $w_{u, v} \geq 0$, a typical transition probability matrix $P$ can be defined as

$$
P_{u v}=\frac{w_{u v}}{\sum_{z} w_{u z}} .
$$

For any initial distribution $f: V \rightarrow \mathbf{R}$, the distribution after one step of a random walk is just

$$
f P(v)=\sum_{u} \frac{w_{u v}}{d_{u}} f(u) .
$$

Here we treat $f$ as a row vector. The distribution after $k$ steps is $f P^{k}(v)$.

In the terminology of Markov chains, a random walk is said to be ergodic if there is a unique stationary distribution $\pi$ satisfying $\pi P=\pi$. Necessary conditions for the ergodicity of a random walk on a graph with $n$ vertices are irreducibility ( i.e., no 0 submatrix of $P$ of size $k \times(n-k)$ for any $k$ ) and aperiodicity, (i.e., g.c.d. $\left.\left\{s: P^{s}(u, v)>0\right\}=1\right)$. As it turns out, these necessary conditions are also sufficient. An ergodic Markov chain is said to be reversable if for any two vertices $u$ and $v$, we have

$$
\pi(u) P(u, v)=\pi(v) P(v, u) .
$$

A reversible Markov chain can be studied as a weighted graph as follows [13]:

THEOREM 18. The following three statements are equivalent:

(a): A Markov chain with transition probability matrix $P$ is ergodic and reversible. 
(b): The weighted graph defined by edge weight $w_{u, v}=\pi(u) P(u, v)$ is connected and non-bipartite.

(c): The weighted graph defined by edge weight $w_{u, v}=\pi(u) P(u, v)$ has one eigenvalue 0 and all other eigenvalues greater than 0 and strictly less than 2 .

It can be easily shown that the stationary distribution $\pi$ satisfies

$$
\pi(v)=\frac{d_{v}}{\operatorname{vol}(G)} .
$$

A natural question of interest is what is the rate of convergence to the stationary distribution. The answer to this question again lies in the eigenvalues of the associated graph.

In the study of rapidly mixing Markov chains, the convergence in the $L_{2}$ distance is rather weak since it it does not require convergence to the stationary distribution at every vertex. A strong notion of convergence that is often used is measured by the relative pointwise distance (r.p.d). After $s$ steps the relative pointwise distance of $P$ to the stationary distribution $\phi(x)$ is given by

$$
\Delta(s)=\max _{x, y} \frac{\left|P^{s}(y, x)-\pi(x)\right|}{\pi(x)}
$$

Another notion of distance for measuring convergence is the so-called it total variation distance:

$$
\begin{aligned}
\Delta_{T V}(s) & =\max _{A \subset V(G)} \max _{y \in V(G)}\left\|\sum_{x \in A}\left(P^{s}(y, x)-\pi(x)\right)\right\| \\
& =\frac{1}{2} \max _{y \in V(G)} \sum_{x \in V(G)}\left\|\left(P^{s}(y, x)-\pi(x)\right)\right\| .
\end{aligned}
$$

It is easy to see that $\Delta_{T V}(s) \leq \frac{1}{2} \Delta(s)$. Thus a convergence upper bound for $\Delta(s)$ implies one for $\Delta_{T V}(s)$.

The rate of convergence of a random walk on a graph on $n$ vertices depends on the spectral gaps, $\lambda_{1}$ and $2-\lambda_{n-1}$. However, the gap $2-\lambda_{n-1}$ can often be circumvented by considering a modified random walk, so- called lazy walk. For a transition probability matrix $P$, a $C$-lazy walk is defined by the transition probablity matrix $P_{C}$, for some $C<1$ :

$$
P_{C}(u, v)= \begin{cases}C+\frac{P(u, u)}{1+C} & \text { if } u=v, \\ \frac{P(u, v)}{1+C} & \text { otherwise. }\end{cases}
$$

The value for $C$ is often chosen to be $1 / 2$. Suppose we choose

$$
C= \begin{cases}0 & \text { if } \lambda_{1} \geq 2-\lambda_{n-1} \\ \frac{\lambda_{1}+\lambda_{n-1}-2}{2} & \text { otherwise. }\end{cases}
$$

Then we have the following:

THEOREm 19. Suppose that a graph $G$ on $n$ vertices has Laplacian eigenvalues $0=\lambda_{0} \leq \lambda_{1} \leq \ldots \leq \lambda_{n-1}$. Then $G$ has a lazy random walk with the rate of convergence of order

$$
\lambda^{-1} \log \left(\frac{\operatorname{vol}(G)}{\min _{x} d_{x}}\right)
$$

where $\lambda=\lambda_{1}$ if $2 \geq \lambda_{1}+\lambda_{n-1}$ and $\lambda=2 \lambda_{1} /\left(\lambda_{1}+\lambda_{n-1}\right)$ otherwise. 
Namely, after at most

$$
t \geq \lambda^{-1}\left(\log \left(\frac{\operatorname{vol}(G)}{\min _{x} d_{x}}\right)+c\right)
$$

steps, we have

$$
\Delta(t) \leq e^{-c} .
$$

For random walks on groups or, equivalently, on graphs defined by groups, the eigenvalues of the Laplacian can often be evaluated exactly using group representation theory. For example, various applications in card shuffling, are associated with graphs with vertex set the symmetric group $S_{n}$ and edge set defined by permutations corresponding to the allowable shuffling moves. There are extensive surveys and books $[\mathbf{3 6}, \mathbf{5 8}]$ on this subject. Here, instead, we consider two applications for general graphs.

Logarithmic Sobolev inequalities The upper bound for the rate of convergence in Theorem 19 can sometimes be further improved by using the log-Sobolev constant $\alpha$ defined as follows [37]:

$$
\alpha(G)=\alpha=\inf _{f \neq 0} \frac{\sum_{x \sim y}(f(x)-f(y))^{2} w_{x, y}}{\sum_{x} f^{2}(x) d_{x} \log \frac{f^{2}(x) \operatorname{vol}(G)}{\sum_{z} f^{2}(z) d_{z}}}
$$

where $f$ ranges over all nontrivial vectors $f: V \rightarrow \mathbb{R}$.

Then we have the following [18]:

THEOREM 20. For a weighted graph $G$ with log-Sobolev constant $\alpha$, there is a lazy walk satisfying

$$
\Delta(t)<e^{2-c}
$$

provided that

$$
t \geq \frac{1}{\alpha} \log \log \frac{\operatorname{vol}(G)}{\min _{x} d_{x}}+\frac{c}{\lambda}
$$

where $\lambda$ is as defined in Theorem 19.

Enumerating contingency tables As an application of the eigenvalue inequalities in Section 9, we consider the classical problem of sampling and enumerating the family $S$ of $n \times n$ matrices with nonnegative integral entries and given row and column sums. Although the problem is presumed to be computationally intractable (in the so-called \#P-complete class), the eigenvalue bounds in the previous section can be used to obtain a polynomial approximation algorithm. To see this, we consider the homogeneous graph $\Gamma$ with the vertex set consisting of all $n \times n$ matrices with integral entries (possibly negative) with given row and column sums. Two vertices $u$ and $v$ are adjacent if $u$ and $v$ differ at just the four entries of a $2 \times 2$ submatrix with entries

$$
u_{i k}=v_{i k}+1, u_{j k}=v_{j k}-1, u_{i m}=v_{i m}-1, u_{j m}=v_{j m}+1 .
$$

The family $S$ of matrices with all nonnegative entries is then a convex subgraph of $\Gamma$.

On the vertices of $S$, we consider the following random walk. The transition probability $P(u, v)$ of moving from a vertex $u$ in $S$ to a neighboring vertex $v$ is $\frac{1}{k}$ if $v$ is in $S$, where $k$ is the degree of $\Gamma$. If a neighbor $v$ of $u$ (in $\Gamma$ ) is not in $S$, then 
we move from $u$ to each neighbor $z$ of $v, z$ in $S$, with the (additional) probability $\frac{1}{d_{v}^{\prime}}$ where $d_{v}^{\prime}=\mid\{z \in S: z \sim v$ in $\Gamma\} \mid$ for $v \notin S$. In other words, for $u, v \in S$,

$$
P(u, v)=\frac{w_{u v}}{d_{u}}+\sum_{\substack{z \notin S \\ u \sim z, v \sim z}} \frac{w_{u z}}{d_{v} d_{z}^{\prime}} w_{z v}
$$

where $w_{u v}$ denotes the weight of the edge $\{u, v\} \quad\left(w_{u v}=1\right.$ or 0 for simple graphs) and $d_{u}=\sum_{u \sim v} d_{u v}$. The stationary distribution for this walk is uniform. Let $\rho$ denote the second largest eigenvalue of $P$. It can be easily checked that $1-\rho \geq \lambda_{1}(S)$, where $\lambda_{1}(S)$ denotes the first Neumann eigenvalue of the induced subgraph $S$ of $\Gamma$. In particular, if the total row sum (minus the maximum row sum) is $\geq c^{\prime} n^{2}$, it can be shown (see $[\mathbf{2 7}]$ ) that $\lambda_{1}(S) \geq \frac{c}{k D^{2}}$, where $D$ is the diameter of $S$. This implies that a random walk converges to the uniform distribution in $O\left(k^{2} D^{2}(\log n)\right)$ steps. (In some cases, the factor $\log n$ can be further reduced by using logarithmic Sobolev inequalities and logarithmic Harnack inequalities (see $[\mathbf{3 7}, \mathbf{1 3}])$.)

It is reasonable to expect that the above techniques can be useful for developing approximation algorithms for many other difficult enumeration problems by considering random walk problems in appropriate convex subgraphs. Further applications using the eigenvalue bounds in previous sections can be found in [31].

\section{Concluding remarks}

1. In this paper, we mainly deal with simple graphs. For a weighted graph $G$ with edge weight $w_{u, v}$, we define $d_{v}=\sum_{u} w_{u, v}$ and $e(X, Y)=\sum_{u \in X, v \in Y} w_{u, v}$. Then the isoperimetric inequalities in Sections 4 and 5 still hold.

2. We consider three families of graphs on $n$ vertices:

$\mathcal{F}_{1}=\{d-$ regular graphs that are $(n, d, \lambda)-$ graphs $\}$,

$\mathcal{F}_{2}=\left\{\right.$ graphs satisfying $\mid$ average degree $-\sigma_{i} \mid \leq \theta$ for $\left.i \neq 0\right\}$,

$\mathcal{F}_{3}=\left\{\right.$ graphs satisfying $\left|1-\lambda_{i}\right| \leq \bar{\lambda}$ for $\left.i \neq 0\right\}$,

where the $\sigma_{i}$ 's are eigenvalues of the combinatorial Laplacian and $\lambda_{i}$ 's are eigenvalues of the normalized Laplacian.

Clearly, we have

$$
\mathcal{F}_{1} \subset \mathcal{F}_{2}
$$

if $\lambda=\theta$. Also

$$
\mathcal{F}_{2} \subset \mathcal{F}_{3}
$$

if $\theta$ is $\bar{\lambda}$ times the average degree. Hence, the isoperimetric inequalities involving eigenvalues of the normalized Laplacian have stronger implications than that of the combinatorial Laplacian. For applications using eigenvalues of the combinatorial Laplacian, it is natural to ask if the same results hold for the normalized Laplacian. For example, is it true that graphs in $\mathcal{F}_{3}$ are Hamiltonian provided that $\bar{\lambda}$ is small enough?

3. Graph theory has 250 years of history. In the very early days, graphs were used to study the structure of molecules and in particular, the eigenvalues of graphs are associated with stability of chemicals $[\mathbf{9}]$. In recent years, many realistic graphs that arise in Internet and biological networks can be modeled as graphs with certain "power law" degree distribution $[\mathbf{1}, \mathbf{2}, \mathbf{3}]$. Again, eigenvalues come into play since random graphs with given expected degrees are shown to have eigenvalue distribution as predicted $[\mathbf{3 2}, \mathbf{3 3}]$. In this paper, we discuss only a few 
applications of isoperimetric inequalities. It would be of interest to find further applications especially for power law graphs.

Acknowledgement: The author wishes to thank Robert Ellis and Josh Cooper for careful reading and making many valuable suggestions for an earlier draft of this paper.

\section{References}

[1] W. Aiello, F. Chung and L. Lu, A random graph model for massive graphs, STOC 2001, 171-180. The paper version appeared in Experimental Math. 10, (2001), 53-66.

[2] W. Aiello, F. Chung and L. Lu, Random evolution in massive graphs, Handbook of Massive Data Sets, Volume 2, (Eds. J. Abello et al.), Kluwer Academic Publishers, (2002), 97-122. Extended abstract appeared in FOCS 2001, 510-519.

[3] R. Albert, H. Jeong and A. Barabási, Diameter of the World Wide Web, Nature 401 (1999), 130-131.

[4] N. Alon, Eigenvalues and expanders, Combinatorica 6, (1986) 86-96.

[5] N. Alon and F. R. K. Chung, Explicit constructions of linear-sized tolerant networks, Discrete Math. 72 (1988), 15-20.

[6] N. Alon, F. R. K. Chung and R. L. Graham, Routing permutations on graphs via matchings, SIAM J. Discrete Math. 7 (1994), 513-530.

[7] N. Alon and V. D. Milman, $\lambda_{1}$ isoperimetric inequalities for graphs and superconcentrators, J. Comb. Theory B 38 (1985), 73-88.

[8] J. Beck, On size Ramsey number of paths, trees, and circuits. I, J. Graph Theory, 7, (19xx), 115-129.

[9] N.L. Biggs, E.K. Lloyd and R.J. Wilson, Graph Theory 1736-1936, Clarendon Press, Oxford, 1976.

[10] B. Bollobás, Extremal Graph Theory, Academic Press, London (1978).

[11] J. Cheeger, A lower bound for the smallest eigenvalue of the Laplacian, Problems in Analysis, (R. C. Gunning, ed.) Princeton Univ. Press (1970), 195-199.

[12] I. Chavel, Isoperimetric inequalities, Differential geometric and analytic perspectives, Cambridge Tracts in Mathematics, 145, Cambridge University Press, Cambridge, 2001.

[13] F. R. K. Chung, Spectral Graph Theory, CBMS Lecture Notes, AMS Publications, 1997.

[14] F. R. K. Chung, Diameters and eigenvalues, J. of Amer. Math. Soc. 2 (1989), 187-196.

[15] F. R. K. Chung, Eigenvalues of graphs and Cheeger inequalities, in Combinatorics, Paul Erdős is Eighty, Volume 2, edited by D. Miklós, V. T. Sós, and T. Szőnyi, János Bolyai Mathematical Society, Budapest (1996), 157-172.

[16] F. R. K. Chung, Universal graphs and induced-universal graphs, J. Graph Theory 14 (1990), 443-454

[17] Fan Chung, A spectral Turán theorem, preprint.

[18] F. R. K. Chung, Logarithmic Sobolev techniques for random walks on graphs, Emerging Applications of Number Theory, IMA Volumes in Math. and its Applications, 109, (eds. D. A. Hejhal et. al.), 175-186, Springer, 1999.

[19] F. R. K. Chung, V. Faber and T. A. Manteuffel, An upper bound on the diameter of a graph from eigenvalues associated with its Laplacian, SIAM J. Discrete Math. 7 (1994), 443-457.

[20] F. R. K. Chung and R. L. Graham, On universal graphs for spanning trees, Journal of London Math. Soc. 27 (1983), 203-211.

[21] F. R. K. Chung, A. Grigor'yan, and S.-T. Yau, Eigenvalues and diameters for manifolds and graphs, Tsing Hua Lectures on Geometry and Analysis, (ed. S.-T. Yau), International Press, Cambridge, Massachusetts, (1997), 79-106.

[22] F. R. K. Chung, A. Grigor'yan, and S.-T. Yau, Upper bounds for eigenvalues of the discrete and continuous Laplace operator, Advances in Mathematics, 117 (1996), 165-178.

[23] F. R. K. Chung, A. Grigor'yan, and S.-T. Yau, Higher eigenvalues and isoperimetric inequalities on Riemannian manifolds and graphs, Communications on Analysis and Geometry, 8 (2000), 969-1026.

[24] F. R. K. Chung and Prasad Tetali, Isoperimetric inequalities for cartesian products of graphs, 7 (1998), 141-148.

[25] F. R. K. Chung and S.-T. Yau, Eigenvalues of graphs and Sobolev inequalities, Combinatorics, Probability and Computing 4 (1995), 11-26. 
[26] F. R. K. Chung and S.-T. Yau, A Harnack inequality for homogeneous graphs and subgraphs, Communications in Analysis and Geometry 2 (1994), 628-639.

[27] F. R. K. Chung and S.-T. Yau, Eigenvalue inequalities of graphs and convex subgraphs, Communications in Analysis and Geometry, 5 (1998), 575-624.

[28] F. R. K. Chung and S.-T. Yau, Logarithmic Harnack inequalities, Mathematical Research Letters 3 (1996), 793-812.

[29] F. R. K. Chung and R. L. Graham, Quasi-random set systems J. Amer. Math. Soc.4 (1991), 151-196

[30] F. R. K. Chung, R. L. Graham and R. M. Wilson, Quasi-random graphs, Combinatorica 9 (1989), 345-362.

[31] F. R. K. Chung, R. L. Graham and S.-T. Yau, On sampling with Markov chains, Random Structures and Algorithms 9 (1996), 55-78.

[32] F. Chung, L. Lu and Van Vu, Eigenvalues of random power law graphs, Annals of Combinatorics, 7 (2003), 21-33.

[33] F. Chung, L. Lu and Van Vu, The spectra of random graphs with given expected degrees, Proceedings of National Academy of Sciences, 100, no. 11, (2003), 6313-6318.

[34] T. Coulhon, A. Grigor'yan and C. Pittet, A geometric approach to on-diagonal heat kernel lower bounds on groups, Ann. Inst. Fourier(Grenoble) 51 (2001), 1763-1827.

[35] T. Coulhon and L. Saloff-Coste, Variétés riemanniennes isométriques à l'infini, Rev. Mat. Iberoamericana, 11 (1995), 687-726.

[36] P. Diaconis, Group representations in probability and statistics, Institute of Mathematical Statistics Lecture Notes - Monograph Series, 11, Institute of Mathematical Statistics, Hayward, CA, 1988.

[37] P. Diaconis and L. Saloff-Coste, Logarithmic Sobolev inequalities for finite Markov chains, Ann. Appl. Probab. 6 (1996), 695-750.

[38] P. Erdős, R. Faudree, C. Rousseau and R. Schelp, On cycle-complete graph Ramsey numbers, J. Graph Theory 2 (1978), 53-64.

[39] J. Friedman and N. Pippenger, Expanding graphs contain all small trees, Combinatorica, 7 (1987), 71-76.

[40] A. Grigor'yan, Heata kernels on manifolds, graphs and fractals, European Congress of Mathematics, vol. 1 (Barcelona, 2000), 393-406, Progr. Math., 201, Birkh auser, Basel, 2001.

[41] M. Jerrum and A. Sinclair, Approximating the permanent, SIAM J. Computing 18 (1989), 1149-1178.

[42] M. Kanai, Rough isometries and combinatorial approximations of geometries of noncompact Riemannian manifolds, J. Math. Soc. Japan 37 (1985), 391-413.

[43] F. Kirchhoff, Über die Aufösung der Gleichungen, auf welche man bei der Untersuchung der linearen Verteilung galvanischer Ströme geführt wird, Ann. Phys. Chem. 72 (1847), 497-508.

[44] J. Komlós amd E. Szemerédi, Limit distributions for the existence of Hamilton circuits in a random graph, Discrete Math. 43, (1983), 55-63.

[45] M. Krivelevich and B. Sudakov, Sparse pseudo-random graphs are Hamiltonian, J. Graph Theory 42 (2003), 17-33.

[46] M. Krivelevich and B. Sudakov, Pseudo-random graphs, preprint.

[47] F. T. Leighton, Introduction to Parallel Algorithms and Architectures: Arrays, Trees, Hypercubes, Morgan-Kauffman, San Mateo, CA, 1992.

[48] F.T. Leighton and Satish Rao, An approximate max-flow min-cut theorem for uniform multicommodity flow problem with applications to approximation algorithms, 29nd Symposium on Foundations of Computer Science, IEEE Computer Society Press, (1988), 422-431.

[49] P. Li and S. T. Yau, On the parabolic kernel of the Schrödinger operator, Acta Mathematica 156, (1986) 153-201

[50] A. Lubotsky, R. Phillips and P. Sarnak, Ramanujan graphs, Combinatorica 8 (1988),261-278.

[51] R. J. Lipton and R.E. Tarjan, A separator theorem for planar graphs, SIAM J. Appl. Math. 36 (1979), 177-189.

[52] G. A. Margulis, Arithmetic groups and graphs without short cycles, 6th Internat. Symp. on Information Theory, Tashkent (1984) Abstracts 1, 123-125 (in Russian).

[53] P. Lancaster, Theory of matrices, Academic Press, 1969.

[54] L. Lovász, Combinatorial Problems and Exercises, North-Holland, 1979.

[55] B. Mohar, Isoperimetric number of graphs, J. of Comb. Theory (B) 47 (1989), 274-291. 
[56] G. Polyá and S. Szegö, Isoperimetric Inequalities in Mathematical Physics, Annals of Math. Studies, no. 27, Princeton University Press, (1951).

[57] L. Pósa, Hamiltonian circuits in random graphs, Discrete Math., 14 (1976), 359-364.

[58] L. Saloff-Coste, Lectures on finite Markov chains, Lectures on Probability Theory and Statistics (Saint-Flour, 1996), Lecture Notes in Math.,1665 Springer, Berlin, (1997), 301-413.

[59] R. M. Tanner, Explicit construction of concentrators from generalized N-gons, SIAM J. Algebraic Discrete Methods 5 (1984), 287-294.

[60] B. Sudakov, T. Szabo and V. Vu, A generalization of Turán's theorem, preprint.

[61] R.M. Tanner, Explicit construction of concentrators from generalized N-gons, SIAM J. Algebraic Discrete Methods 5 (1984), 287-294.

[62] V. Vu, A general upper bound on the list chromatic number of locally sparse graphs, Combinatorics, Probability and Computing 11 (2002), 103-111.

[63] W. Woess, Random walks on infinite graphs and groups, Cambridge Tracts in Mathematics, 138, Cambridge University Press, Cambridge, 2000.

[64] S. T. Yau and R. M. Schoen, Differential Geometry, (1994), International Press, Cambridge, Massachusetts.

University of California at San Diego, La Jolla, CA 92093-0112, USA

E-mail address: fan@euclid.ucsd.edu 